\title{
Effect of cocoa on the brain and gut in healthy subjects: a randomised controlled trial
}

\author{
Mark Fox ${ }^{1,2 *}$, Anne Christin Meyer-Gerspach ${ }^{3}$, Maria Janina Wendebourg ${ }^{1,4}$, Maja Gruber ${ }^{1}$, \\ Henriette Heinrich $^{1,2}$, Matthias Sauter ${ }^{1,2}$, Bettina Woelnerhanssen ${ }^{3}$, Dieter Koeberle ${ }^{5}$ and \\ Freimut Juengling 5 \\ ${ }^{1}$ Abdominal Center: Gastroenterology, St. Claraspital, CH-4058 Basel, Switzerland \\ ${ }^{2}$ Department of Gastroenterology and Hepatology, University Hospital Zürich, CH-8091 Zürich, Switzerland \\ ${ }^{3}$ St. Clara Research Ltd, St. Claraspital, CH-4058 Basel, Switzerland \\ ${ }^{4}$ Department of Neurology, University Hospital Basel, CH-4058 Basel, Switzerland \\ ${ }^{5}$ Department of Nuclear Medicine and PET/CT-Center North-West Switzerland, St. Claraspital, CH-4058 Basel, Switzerland
}

(Submitted 3 October 2018 - Final revision received 22 November 2018 - Accepted 5 December 2018 - First published online 24 January 2019)

\begin{abstract}
Dark chocolate is claimed to have effects on gastrointestinal function and to improve well-being. This randomised controlled study tested the hypothesis that cocoa slows gastric emptying and intestinal transit. Functional brain imaging identified central effects of cocoa on cortical activity. Healthy volunteers (HV) ingested $100 \mathrm{~g}$ dark ( $72 \%$ cocoa) or white ( $0 \%$ cocoa) chocolate for $5 \mathrm{~d}$, in randomised order. Participants recorded abdominal symptoms and stool consistency by the Bristol Stool Score (BSS). Gastric emptying (GE) and intestinal and colonic transit time were assessed by scintigraphy and marker studies, respectively. Combined positron emission tomography-computed tomography (PETCT) imaging assessed regional brain activity. A total of sixteen HV (seven females and nine males) completed the studies (mean age 34 (21-58) years, BMI $22.8(18.5-26 \cdot 0) \mathrm{kg} / \mathrm{m}^{2}$ ). Dark chocolate had no effect on upper gastrointestinal function (GE half-time 82 (75-120) $v .83$ (60120) min; $P=0.937$ ); however, stool consistency was increased (BSS $3(3-5) v .4(4-6) ; P=0.011$ ) and there was a trend to slower colonic transit (17 (13-26) v. $21(15-47) \mathrm{h} ; P=0 \cdot 075)$. PET-CT imaging showed increased $\left[{ }^{18} \mathrm{~F}\right]$ fluorodeoxyglucose (FDG) in the visual cortex, with increased FDG uptake also in somatosensory, motor and pre-frontal cortices $(P<0 \cdot 001)$. In conclusion, dark chocolate with a high cocoa content has effects on colonic and cerebral function in HV. Future research will assess its effects in patients with functional gastrointestinal diseases with disturbed bowel function and psychological complaints.
\end{abstract}

Key words: Chocolate: Cocoa: Gastric emptying: Colon transit time: $\left[{ }^{18}\right.$ F]fluorodeoxyglucose-positron emission tomography

Already in the time of the Mayas and Aztecs, the fruit of the cocoa tree (Theobroma cacao) was used as medicine; and, still today, popular claims confer on this 'food of the gods' properties of being a stimulant, relaxant, aphrodisiac and antidepressant. The difference between cocoa and chocolate is that cocoa is the non-fat component of cocoa liquor (finely ground cocoa beans), but chocolate contains a combination of ingredients that include cocoa, cocoa butter, sugar and other constituents formed into a solid food. Dark chocolate with a high cocoa content contains flavonoids (e.g. epicatechin), methylxanthines (e.g. caffeine, theobromine) and other compounds with antioxidant and other potentially health-promoting properties $^{(1,2)}$. In contrast, white chocolate contains very few biologically active substances but no $\operatorname{cocoa}^{(3,4)}$. Recent studies suggest that, despite its high-fat and -energy content, regular intake of dark chocolate has beneficial effects on various markers of metabolic health and is associated with reduction in the risk of heart attack, stroke and dementia ${ }^{(5-9)}$.

Dark chocolate is also thought to have effects on gastrointestinal function and is traditionally used as a home remedy for diarrhoea ${ }^{(10)}$; however, objective evidence for the impact of cocoa on the gastrointestinal time or symptoms is lacking. Similarly, although chocolate has hedonic appeal and a positive influence on $\operatorname{mood}^{(11,12)}$, the central mechanisms behind these effects remain uncertain.

There is increasing interest in the effects of diet on the brain and the gut in health and disease, in particular functional gastrointestinal diseases such as irritable bowel syndrome (IBS). Randomised controlled trials indicate that IBS patients benefit from medical treatment targeting digestive function (e.g.

Abbreviations: HV, healthy volunteer; PET-CT, positron emission tomography-computed tomography; VAS, visual analogue scale.

* Corresponding author: M. Fox, Department of Gastroenterology and Internal Medicine, Klinik Arlesheim, CH-4144 Arlesheim, Switzerland, email dr.mark.fox@gmail.com 
regulation of stool habit) and neuropsychological state (e.g. effects of antidepressants on visceral sensitivity and mood) ${ }^{(13-15)}$. However, many IBS patients prefer dietary and other 'natural remedies' to the regular intake of pharmaceuticals ${ }^{(16)}$. The evidence referred to above suggests that dark chocolate may have 'nutraceutical' effects on the brain and the gut that could benefit individuals with disorders of gastrointestinal motor and sensory function.

Ahead of clinical trials in patients, this research tested the hypothesis that dark chocolate with $72 \%$ cocoa content would slow gastrointestinal transit (primary outcome) and have specific effects on regional brain activity in healthy volunteers (HV). The study was performed using dark chocolate and not cocoa powder or biologically active components extracted from cocoa because it is not known which, if any, components of this product have the desired effects on the brain and the gut. A prospective, counterbalanced, crossover study was performed in HV. Subjects ingested either $100 \mathrm{~g}$ of dark or white chocolate (control with $0 \%$ cocoa content) for five consecutive days in randomised order with a minimum 2-week wash out period between interventions. Validated methods were applied to assess the effects of the test substances on abdominal symptoms, gastric emptying, oro-caecal and colonic transit times. In addition, we looked for the effects on regional brain activity using combined positron emission tomography-computed tomography (PET-CT) imaging. The results provide novel evidence concerning the effects of chocolate on the gut and the brain.

\section{Methods}

\section{Participants}

Adult HV aged 18-65 were recruited by advertisement. Participants had no indication of medical or psychological disease, no history of abdominal surgery (other than appendicitis or hysterectomy) and no pathology on physical examination. Subjects were excluded if they had a BMI of $<18$ or over $30 \mathrm{~kg} / \mathrm{m}^{2}$, took medication which may affect gastric motility, had an eating disorder, had vegan diet or were allergy to milk protein or cocoa.

All participants reported eating chocolate regularly. According to industry statistics (https://www.chocosuisse.ch/en/services4/facts-figures-4-2/), the average chocolate consumption in Switzerland is approximately $10 \mathrm{~kg} /$ year (equating to $25 \mathrm{~g} / \mathrm{d}$ ); however, this includes white, milk and dark chocolate. This indicates that the dose provided in the dark chocolate dietary intervention is at least four times the normal daily cocoa intake in this population.

The study protocol conforms to the ethical guidelines of the 1975 Declaration of Helsinki as reflected in a priori approval by the Ethics Committee of North-West Switzerland (Reference: 2016-01841 (21.12.2016)). Approval was also granted by the local radiological protection board. The study was registered (ClinicalTrials.gov, NCT03022955, https://register.clinicaltrials.gov/ (10 January 2017)). Written informed consent was obtained from each participant.

\section{Protocol}

Subjects were assigned to receive either dark (Chocolat Frey Noir Spezial ( $72 \%$ cocoa solids)) or white (Chocolat Frey Blanca (0\% cocoa solids)) chocolate on five consecutive days in a randomised order. According to the information on file, the dark chocolate used in this study contains $43 \mathrm{mg}$ caffeine and $250 \mathrm{mg}$ flavanols (measured by HPLC). White chocolate contains no caffeine and no flavanols. Alkali extraction was not used for these products (process reduces flavanol concentration).

Block randomisation in groups of four was performed to ensure all participants tested on any $1 \mathrm{~d}$ received the same meal with the order of conditions counterbalanced, so that $50 \%$ started with dark chocolate. The two study periods were separated by a minimum of $14 \mathrm{~d}$ washout. Subjects were instructed to continue with their normal diet and lifestyle throughout (a food diary was not required). Intake of additional chocolate during the dietary intervention was not allowed. Failure to take the dark or white chocolate was also an exclusion criterion. All physiological investigations were completed in St. Claraspital between 3 February and 20 May 2017. Participants were not blinded to the dietary intervention (white or dark chocolate); however, those taking part were not aware of the study hypothesis or that white chocolate was the control condition. Further, investigators analysing the data were blinded to the test substance and meal. Each study period lasted $5 \mathrm{~d}$. On days 1 to 3 subjects ingested a chocolate bar (both $100 \mathrm{~g}$, $2100 \mathrm{~kJ}, 50 \%$ fat) with a capsule containing ten radio-opaque markers for assessment of colonic/whole-gut transit time. On day 4 and 5, the same amount of cocoa was ingested as a mousse (total $150 \mathrm{~g}, 2100 \mathrm{~kJ}$ (online Supplementary Table S1)). On day 4 , ingestion of the chocolate was followed by a combined PET-CT brain scan (see below) and a low-intensity CT of the abdomen to assess colonic/whole-gut transit time. On day 5, a non-absorbable Technecium-99m-DTPA marker was added to the mousse for scintigraphic imaging of the test meal (estimated $19 \mathrm{mSv}$ exposure for complete study).

\section{Gastrointestinal scintigraphy, well-being and postprandial sensation}

Anterior and posterior planar images were acquired before and every $15 \mathrm{~min}$ for $120 \mathrm{~min}$ after ingestion of labelled chocolate mousse by a Gamma Camera (Nucline X-Ring-R; Mediso). After each scan, the subjects were asked to score satiety, fullness, bloating, heartburn, nausea and epigastric pain using a validated $100 \mathrm{~mm}$ visual analogue scale $(\mathrm{VAS})^{(17)}$. Sensations were rated as follows: mild $<30$, mild to moderate 30-60, moderate to severe 60-90 and severe $>90 \mathrm{~mm}$. Less than severe fullness and satiety are considered normal postprandial sensations. More than mild bloating, nausea, abdominal pain and heartburn are pathological 'dyspeptic' sensations ${ }^{(17)}$.

Gastric emptying half-time, gastric retention at $120 \mathrm{~min}$ and oro-caecal transit time were assessed using validated protocols $^{(18)}$. Measurements were performed by three independent researchers (H. H., M. S., M. F.) without knowledge of patient allocation and the mean value calculated.

\section{Bristol Stool Score and colonic/whole-gut transit time}

The subjects recorded stool consistency using the validated Bristol Stool Score on a scale of 1 (very hard) to 7 (liquid) as 
illustrated by Fig. $4^{(19)}$. Objective measurements were determined by counting the number of radio-opaque markers retained in the colon. A simple conversion factor was applied to calculate the colonic transit time ${ }^{(18)}$.

\section{Functional brain imaging}

The right-handed HV were assigned to brain imaging studies using a crossover design for the digestion of either cocoa-rich dark or cocoa-free white chocolate mousse. Methods were identical for both study arms. PET-CT was preferred to functional MRI because the relatively long acquisition time increases test sensitivity and is less subject to variation in the concentration of biologically active substances in cocoa in the blood stream over time (the optimal time for acquisition of brain imaging after ingestion of cocoa flavanols is uncertain) ${ }^{(12)}$. Subjects were seated in a relaxing chair and an intravenous line was placed. Chocolate mousse was ingested from a bowl using a standard spoon within $10 \mathrm{~min}$. After meal commencement (two spoons), $100 \mathrm{MBq}\left[{ }^{18} \mathrm{~F}\right]$ fluorodeoxyglucose (FDG) was injected while the subjects continued ingesting the mousse to completion. Directly after completing the meal (i.e. imaging did not begin during meal ingestion), subjects were transferred to a Siemens Biograph 40 HIREZ TRUE-D whole-body PET-CT (Siemens Medical Systems). Brain scans were acquired in a brightly lit facility. All patients were awake during measurements; however, alertness was not monitored. After low-dose CT for attenuation correction and anatomical correlation, a pseudodynamic PET acquisition (five timeframes at 4 min each) was started and continued for maximum $2 \mathrm{~h}$. Images were reconstructed using a point spread function recovering iterative reconstruction (HD-PET (tm) Siemens Medical Systems) resulting in reconstructed images of $2 \mathrm{~mm}$ isotropic resolution.

Emission data were reconstructed to generate an image of relative glucose metabolism, as described previously ${ }^{(20)}$. Briefly, attenuation corrected images were analysed using the generalised linear model as implemented in SPM12 (Wellcome Trust Centre for Neuroimaging, UCL) and a voxel-wise ANCOVA between the two study conditions was performed with correction for potential confounding covariates (e.g. age, sex $)$ for each subject and session $(P<0.001$ corrected for multiple comparisons using the field wise error correction were regarded as significant). For the purpose of visualisation, activated voxel clusters were projected on a three-dimensional imaging atlas brain as provided by SPM12, and their centre of activation reported as coordinates within the stereotactic standard space of the Montreal Neurologic Institute brain atlas.

\section{Statistical analysis}

Previous studies have demonstrated mean gastric emptying half-time (T50) of $49 \mathrm{~min}$ with test-retest variability $\pm 8 \mathrm{~min}$ for scintigraphic measurements of a $400 \mathrm{ml}, 1250 \mathrm{~kJ}$ nutrient liquid meal $^{(21)}$. Power calculations show twelve paired comparisons are needed to detect a statistically significant difference in T50 (primary outcome) exceeding the daily variability ( $\beta 90 \%, \alpha$ of 0.05). We included sixteen subjects in the study to facilitate assessment also of secondary and exploratory outcome parameters (e.g. colonic transit, brain imaging data). The crossover study design was appropriate to compensate for wide interindividual variation in digestive and cerebral function. In this early phase study, a correction for multiple comparisons was not applied. The study was not powered to identify interactions between the brain and the gut. Demographic and physiological results showed non-parametric distribution and are reported as median with either range or interquartile range as appropriate. Within-subject difference in parameters between the two test substances was investigated with the Wilcoxon paired tests.

\section{Results}

\section{Participants}

After initial telephone screening, nineteen subjects were interviewed and consented. Three withdrew before test procedures being performed. A total of sixteen HV (seven females, nine males) with mean age 34 (21-58) years and mean BMI $22 \cdot 8$ $(18.5-26 \cdot 0) \mathrm{kg} / \mathrm{m}^{2}$ completed studies. Three female participants were taking an oral contraceptive and one had a coil in situ. One male patient was taking an oral anticoagulant (Xarelto) due to an uncomplicated lower limb venous thrombosis. At screening no subject had a psychological disorder (Hospital Anxiety and Depression Scale $<11$ ) and all reported excellent gastrointestinal well-being ( $>75 / 100$ VAS). All subjects tolerated the complete study protocol, with no side effects.

At screening, ten participants reported a preference for dark chocolate with four preferring white chocolate and two stating no preference. After completion of each study arm, palatability of the dark and white chocolate was assessed and was similar (median VAS $62 v$. 69/100; $P=0 \cdot 859$ ).

\section{Well-being and postprandial sensation}

Participants reported no change in well-being after ingestion of dark and white chocolate (73 (63-100) v. 73 (66-93) mm VAS; $P=0.951)$. After completing the chocolate mousse meals, subjects reported an increase in fullness and satiety (both $P<0 \cdot 001$ ) which was similar for dark and white chocolate (postprandial fullness: 58 (40-95) v. 60 (48-95) mm VAS; $P=0 \cdot 323$ ). Subsequently, as emptying progressed, there was a decline in both sensations (Fig. 1). Mild to moderate bloating ( $>30 \mathrm{~mm}$ but $<60 \mathrm{~mm}$ VAS) was reported by one (6\%) subject. No other symptoms were reported.

\section{Gastric emptying and oro-caecal transit time}

Gastric emptying of the semi-solid, nutrient meal was approximately linear. Emptying half time was not different after ingestion of $2100 \mathrm{~kJ}$ dark or white chocolate mousse (82 (75-120) v. 83 (60-120) min; $P=0.937)$ with a similar finding for gastric retention at $120 \mathrm{~min}(32(27-63)$ v. $33(23-57) \%$; $P=0.585)$.

Oro-caecal transit time was also near identical for both test meals (59 (45-98) v. 59 (53-83) min; $P=0.907)$. Note that gastric emptying was rapid in the early and then slowed in 

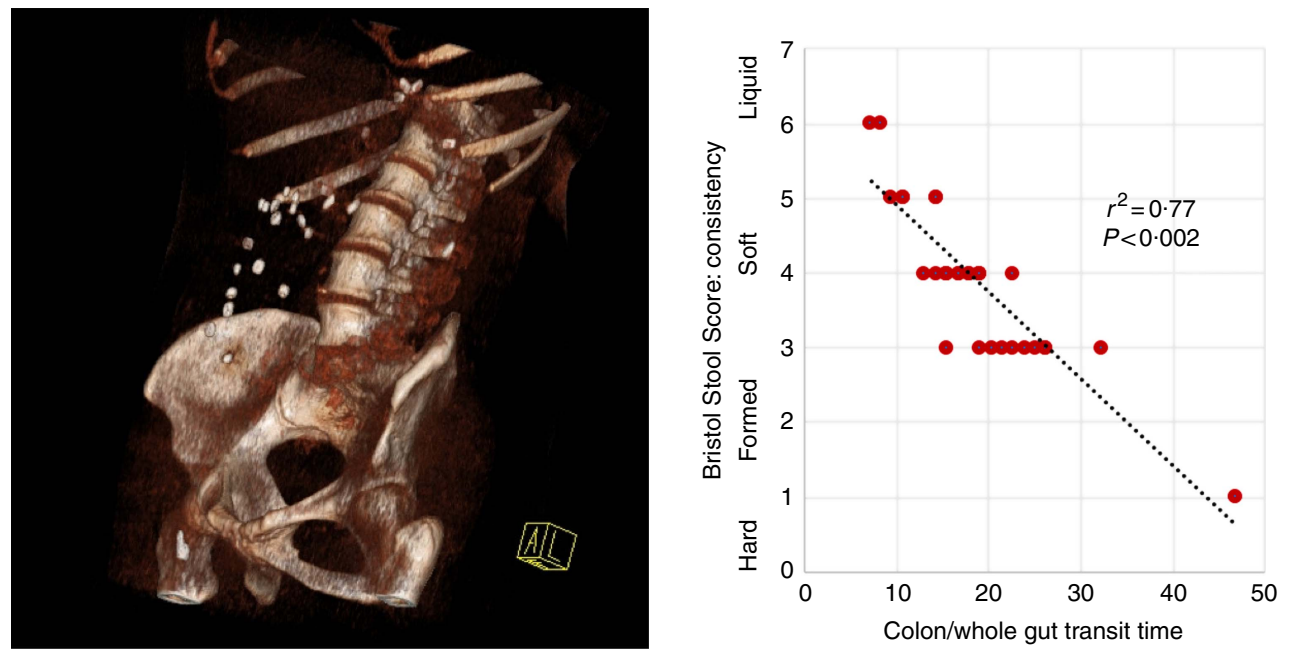

Fig. 1. Three-dimensional reconstruction of low-intensity computed tomography image illustrating assessment of colonic/whole-gut transport by radio-opaque markers (left panel). A number of markers are visible within the ascending and transverse colon. There is a relationship between transit time and stool consistency, such that the longer the stool is retained in the colon, the more water is absorbed and the harder it becomes (right panel).
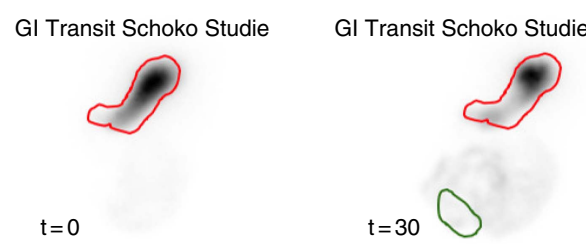

GI Transit Schoko Studie
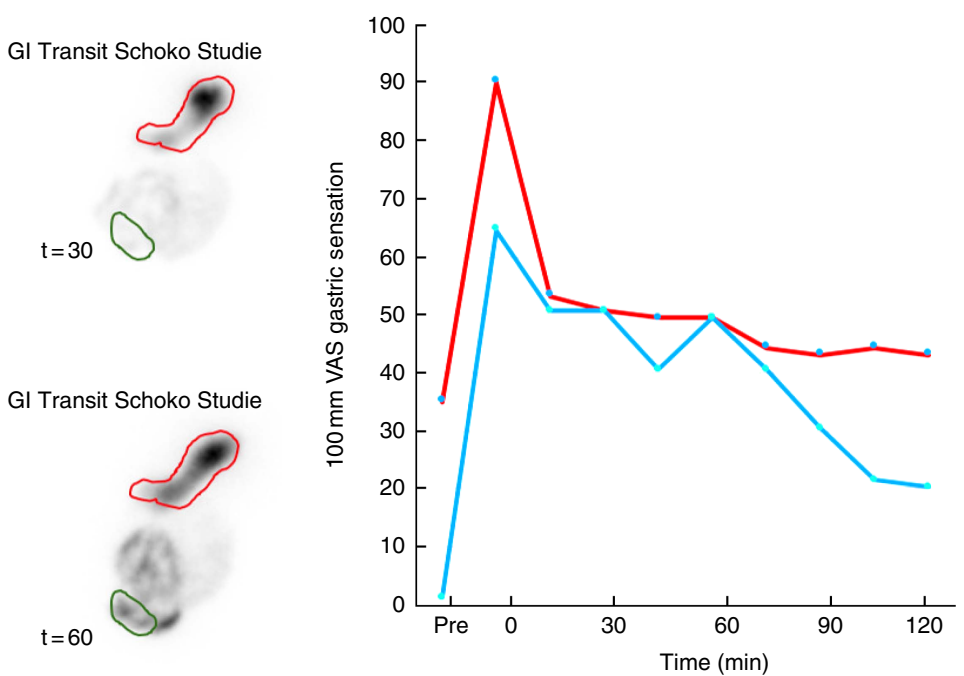

Fig. 2. Representative data illustrating gastrointestinal (GI) motor and sensory function after ingestion of a $2100 \mathrm{~kJ}$ chocolate mousse. Gastric emptying and orocaecal transit (left panel) are measured with reference to gastric (red) and caecal (green) regions of interest. There is initial filling of the proximal stomach by the semisolid meal. Gastric emptying progresses at a steady rate and is accompanied by a steady decrease in fullness and satiety (right panel). After 45 min, more than $50 \%$ of the meal is still in the stomach but contrast is clearly visible in the caecum. ——, Satiety; ——, fullness; VAS, visual analogue scale.

the late postprandial phase, such that contrast is visible in the caecum well before the stomach has half emptied (Fig. 1).

\section{Bristol Stool Scale and colonic/whole-gut transit time}

Participants reported higher stool consistency after 3-d ingestion of dark compared with white chocolate (Bristol Stool Score $3(3-5)$ v. 4 (4-6); $P=0 \cdot 011)$. Consistent with this finding, objective measurement of colonic/whole-gut transit time showed a trend to slower transit after ingestion of dark chocolate $(17(13-26) v .21(15-47) \mathrm{h} ; P=0.075)$, and there was a significant negative correlation (Fig. 2) between the stool score and the transit time (Pearson correlation coefficient $r^{2}-0.77$; $P<0 \cdot 002)$.

\section{Functional brain imaging}

Comparing the spontaneous steady-state cerebral glucose metabolism of dark chocolate intake $v$. white chocolate, the PET-CT imaging showed a highly significant increase in regional glucose metabolism in a large cluster of voxels in the occipital and visual cortex as well as in more circumscribed clusters located in the somatosensory cortex, motor and prefrontal cortices (Fig. 3). All voxel clusters showed highly significant effects both on the level of cluster and peak intensity analysis (both $P<0 \cdot 001$, corrected for multiple comparisons).

\section{Discussion}

The results of this randomised controlled study confirm that dark chocolate with $72 \%$ cocoa content has distinct effects on 

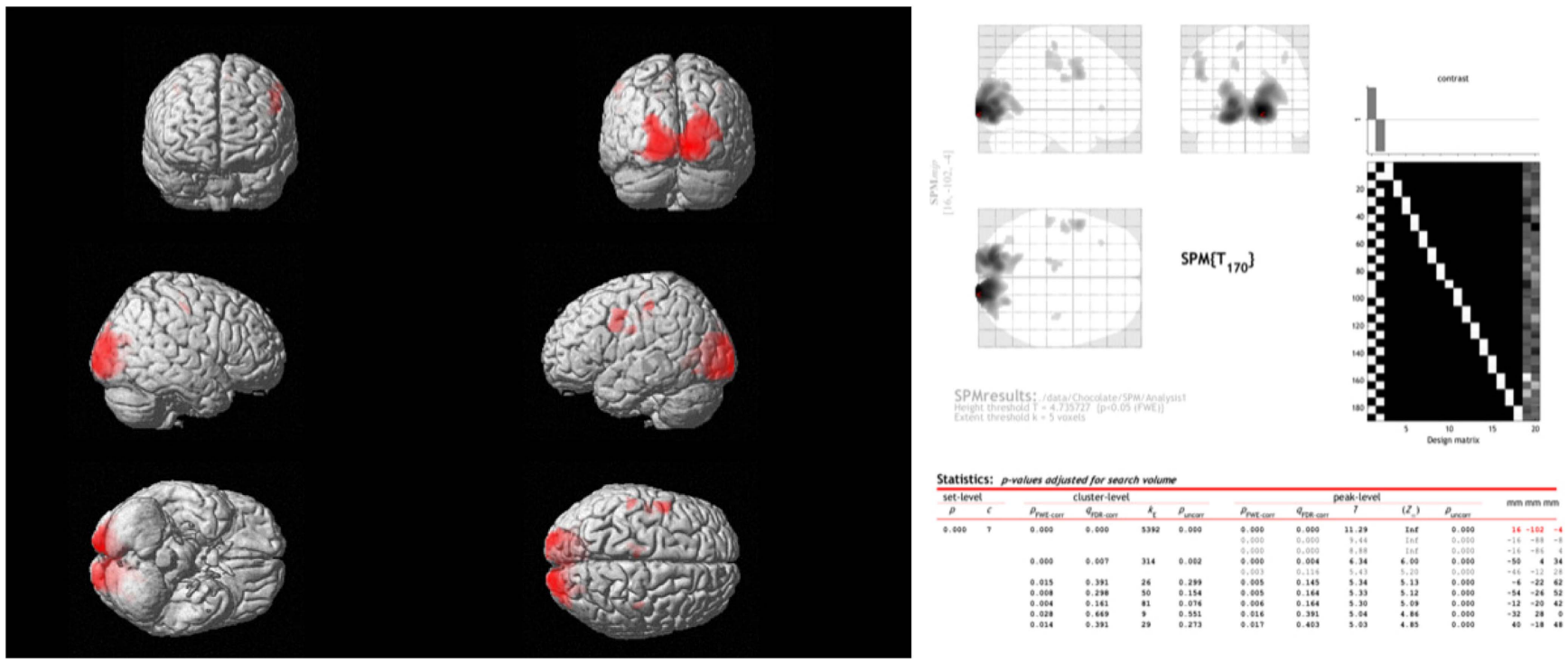

Fig. 3. Combined positron emission tomography (PET)-computed tomography data (left panel) reconstructed to generate an image of relative glucose metabolism. Ingestion of dark chocolate with high cocoa content increases $\left[{ }^{18} \mathrm{~F}\right]$ fluorodeoxyglucose uptake in the visual cortex, with increased activity also in areas of the somatosensory cortex (possibly involved in gustation), motor and pre-frontal cortices (both $P<0.001$ ). Averaged PET data with detailed results (right panel) highlight the differences in brain activation after ingestion of dark and white chocolate.

the brain and the gut compared with the white chocolate control. An effect on psychological well-being was not observed; however, this is not surprising as the HV studied had no evidence of anxiety or depression. Nevertheless, since hedonic factors may affect cerebral and gastrointestinal function, it is important to note that participants rated both test substances as similarly pleasurable to eat.

\section{Gastrointestinal function}

A series of measurements, illustrated by Figs. 1 and 2, documented gastrointestinal transit from ingestion to evacuation. Gastric emptying (primary outcome measure) and oro-caecal transit time were almost identical after ingestion of dark or white chocolate mousse. These results add to the evidence that the key determinants of gastric function are the volume and energy content of meals ${ }^{(22)}$. The delivery of carbohydrates, protein and fat into the small bowel triggers the release of peptide hormones that regulate the delivery of food into the small intestine. However, with the exception of alcohol $^{(23)}$, there is little evidence that specific foods have an important impact on gastric emptying in controlled studies $^{(24)}$.

Participants also reported similar levels of fullness and satiety after the two meals. These sensations increased after ingestion of the chocolate mousse and then returned towards normal levels as gastric emptying proceeded (Fig. 1). The HV rarely reported dyspeptic symptoms after completing the high fat ( $50 \%)$, high energy $(2100 \mathrm{~kJ})$ meal. This could be different in participants with functional gastrointestinal symptoms. More than other nutrients, fat increases the sensation of fullness, nausea and other symptoms in laboratory and clinical studies $^{(25-28)}$. For example, the results of ambulatory $\mathrm{pH}$ studies in patients referred for investigation of suspected gastrooesophageal reflux disease show that, independent of the number of reflux events, there was a $40 \%$ reduction in reflux symptoms reported after low-fat, high-energy compared with high-fat, high-energy meals ${ }^{(29)}$.

In contrast, significant effects of dark chocolate with $72 \%$ cocoa content were observed on the lower gastrointestinal tract. Participants reported a significant increase in stool consistency as illustrated by the Bristol Stool Score. At the same time, there was a clear trend to slower colonic transit documented by radioopaque markers swallowed every day with the dark chocolate bars compared with the control. These findings are consistent with the widely held view that dark chocolate can be constipating $^{(10)}$.

The presence of naturally occurring methylxanthines in dark chocolate did not counteract this effect. High levels of caffeine such as found in a cup of strong brewed coffee (typically $80-150 \mathrm{mg}$ in a regular serving (8 oz; approximately $235 \mathrm{ml}$ )) can trigger the gastro-colonic reflex and accelerate colonic transit ${ }^{(30)}$; however, lower levels found in dark chocolate may not trigger this response ${ }^{(31)}$. One potential mechanism for the inhibitory effect of cocoa on colonic function is the inhibition of chloride channels by cocoa flavonoids, leading to reduced water transport across the colonic epithelium ${ }^{(32)}$. Another possibility is that bioactive substances found in cocoa may antagonise digestive enzymes or exert prebiotic effects on the gut microbiota ${ }^{(33)}$. There is a growing literature that describes striking bidirectional associations between diet, intrinsic gut microbes and the brain, including a role in regulating response to stress and psychological state ${ }^{(34)}$. If proven, it may turn out that dark chocolate not only feeds us but also feeds the billions of microorganisms living in our bowels and that this arrangement is good for both parties! 


\section{Functional brain imaging}

Compared with ingestion of white chocolate, ingestion of dark chocolate with a high cocoa content had no effect on the activity of brain regions responsible for controlling emotions or signalling reward such as the limbic cortex or amygdala. This is consistent with the prevailing view that the mood-enhancing effects of chocolate are not caused by naturally occurring psychoactive substances (e.g. anandamide, an agonist of endogenous cannabinoid receptors) but are more likely explained by properties shared by both dark and white chocolate such as satisfaction of hunger and pleasurable oro-sensory qualities including high palatability, sweetness and optimal mouthfeel $^{(12)}$.

Instead, PET-CT imaging revealed specific effects of dark chocolate on regional glucose metabolism within the occipital and visual cortex, with smaller areas of increased brain activity also in areas of the somatosensory cortex (possibly involved in gustation), motor and pre-frontal cortices (Fig. 3). This result is unlikely to be due to 'visual exposure' to the different test meals. Although the radioactive marker (FDG) was injected during ingestion of the white and dark chocolate mousse, measurements commenced only after completion of the meal and were obtained in a closed scanner with little visual stimulation. It is more likely that biologically active substances present in cocoa had effects on regional brain activity. Flavanols have effects on vascular endothelium that increase peripheral, cardiac and cerebral blood flow via dose-dependent increases in flow-mediated vasodilation ${ }^{(35,36)}$. Controlled studies performed by experimental psychologists indicate consumption of high-dose cocoa flavanols (250-1000 mg) improves a variety of cognitive tasks, including visual contrast sensitivity, time to detect motion direction and spatial working memory performance ${ }^{(37-39)}$. Since increased delivery of substrates improves the performance of mentally effortful tasks, the findings of the PET-CT study may indicate that improved performance of visual information processing is related to effects of cocoa flavanols on the perfusion and regional metabolism of the visual cortex and other higher centres. It is less likely that the results are mediated by methylxanthines in dark chocolate. Caffeine and to a lesser extent theobromine increase alertness, especially in subjects with fatigue, and this is associated with activity in regions involved in the control of vigilance, anxiety and cardiovascular regulation ${ }^{(12,31)}$; however, these substances do not have established effects on the areas identified in this study.

To our knowledge, this PET-CT study is the first to assess the baseline brain glucose metabolism at a steady state after a meal of flavanol-rich dark $v$. flavanol-absent white chocolate mousse preceded by $5 \mathrm{~d}$ intake of the respective 'chocolate bars'. An effect of flavanol-rich cocoa on cerebral blood flow has also been reported by functional MRI studies ${ }^{(40)}$; however, these experiments measured brain activation during cognitive tasks within the scanner and, not surprisingly, different regions of the brain were activated. Further studies are required to establish whether improvements in visual and cognitive performance are directly linked to the effects on brain activity induced by cocoa documented by PET-CT measurements.

\section{Limitations}

This study in sixteen HV has a robust randomised, controlled study design; however, it was not double-blinded, since the control treatment for dark chocolate was white chocolate, and no attempt was made to mask the visual or gustatory differences between the products. Lack of blinding could introduce bias; however, subjects were not aware of the study hypothesis or that white chocolate was the control condition. Further, investigators analysing the data were blinded to the test substance and meal.

In addition, it is not possible to determine which biologically active component of cocoa had effects on the digestive and neurological systems. For example, the two treatments differed not only in flavanol but also in caffeine content. As discussed above, although caffeine alone cannot explain the observations, the possibility that caffeine, theobromine or other components of cocoa (such as $\mathrm{Mg}$ ) interact differentially with flavanols to produce the biological effects of chocolate cannot be ruled out.

\section{Conclusion and potential application in clinical practice}

This dietary intervention study in HV identified specific effects of dark chocolate on digestive and cerebral function. These findings add to the body of literature indicating that naturally occurring substances in chocolate, in particular cocoa flavanols, in isolation and in combination, have measurable biological effects. Future research will establish whether these are clinically relevant. Acute and chronic disturbances in bowel function are common in the community and can be a major burden ${ }^{(41)}$. Drugs used in the therapy of diarrhoea (e.g. loperamide) are not always effective and may cause side effects, leading to decreased acceptance by patients ${ }^{(42)}$. Moreover, many doctors prefer to avoid the use of opioid-based medicines for acute infectious diarrhoea or for the long-term control of chronic diarrhoea. This position is shared by many individuals with functional gastrointestinal diseases that often report side effects with medications and prefer 'natural' remedies ${ }^{(16)}$. If the 'nutraceutical' effects of dark chocolate or cocoa-based products are confirmed, then this could be a safe and popular alternative to pharmacological treatment. If, as expected, there are also positive effects on well-being, then the combination of peripheral and central effects could be a much needed, novel approach for the treatment of participants with conditions such as IBS that are affected by both disorders of digestive function and mood.

\section{Acknowledgements}

The authors would like to thank Bastian Trimpin for performance of the nuclear medical investigations, Jennifer Wagner for expert nutritional advice and Hermann Fritz for preparation of the excellent chocolate mousse!

This study was supported by research awards from Chocosuisse, the association of Swiss chocolate manufacturers and Laboratoire Viollier, Allschwil, Switzerland. Chocolate bars were provided free of charge by Chocolat Frey, Buchs, Switzerland. 
Chocosuisse, Laboratoire Viollier as well as Chocolat Frey had no role in the design, analysis or writing of this article.

M. F., D. K. and F. J. developed the study concept and protocol. M. F., A. C. M.-G., M. J. W. and B. W. were involved in planning and performance of study with data collection. M. G., H. H. and M. S. were involved in data collection and analysis; M. F. directed data analysis and interpretation and wrote the manuscript. M. F. has primary responsibility for the final content. All authors approved the final version of the manuscript.

The authors have no conflicts of interest to declare, except their love for chocolate.

\section{Supplementary material}

For supplementary material/s referred to in this article, please visit https://doi.org/10.1017/S0007114518003689

\section{References}

1. Visioli F, Bernaert H, Corti R, et al. (2009) Chocolate, lifestyle, and health. Crit Rev Food Sci Nutr 49, 299-312.

2. Katz DL, Doughty K \& Ali A (2011) Cocoa and chocolate in human health and disease. Antioxid Redox Signal 15, 2779-2811.

3. Lippi G, Franchini M, Montagnana M, et al. (2009) Dark chocolate: consumption for pleasure or therapy? J Thromb Thrombolysis 28, 482-488.

4. Corti R, Perdrix J, Flammer AJ, et al. (2010) Dark or white chocolate? Cocoa and cardiovascular health. Rev Med Suisse 6 , 499-500, 502-504.

5. Larsson SC, Virtamo J \& Wolk A (2012) Chocolate consumption and risk of stroke: a prospective cohort of men and metaanalysis. Neurology 79, 1223-1229.

6. Grassi D, Desideri G \& Ferri C (2013) Protective effects of dark chocolate on endothelial function and diabetes. Curr Opin Clin Nutr Metab Care 16, 662-668.

7. Grassi D, Socci V, Tempesta D, et al. (2016) Flavanol-rich chocolate acutely improves arterial function and working memory performance counteracting the effects of sleep deprivation in healthy individuals. J Hypertens 34, 1298-1308.

8. Alkerwi A, Sauvageot N, Crichton GE, et al. (2016) Daily chocolate consumption is inversely associated with insulin resistance and liver enzymes in the Observation of Cardiovascular Risk Factors in Luxembourg study. Br J Nutr 115 1661-1668.

9. Larsson SC, Akesson A, Gigante B, et al. (2016) Chocolate consumption and risk of myocardial infarction: a prospective study and meta-analysis. Heart 102, 1017-1022.

10. Muller-Lissner SA, Kaatz V, Brandt W, et al. (2005) The perceived effect of various foods and beverages on stool consistency. Eur J Gastroenterol Hepatol 17, 109-112.

11. Bruinsma K \& Taren DL (1999) Chocolate: food or drug? J Am Diet Assoc 99, 1249-1256.

12. Scholey A \& Owen L (2013) Effects of chocolate on cognitive function and mood: a systematic review. Nutr Rev 71, 665-681

13. Fox MR, Kahrilas PJ, Roman S, et al. (2018) Clinical measurement of gastrointestinal motility and function: who, when and which test? Nat Rev Gastroenterol Hepatol 15, 568-579.

14. Ford AC, Talley NJ, Spiegel BM, et al. (2008) Effect of fibre, antispasmodics, and peppermint oil in the treatment of irritable bowel syndrome: systematic review and meta-analysis. BMJ 337, a2313.
15. Ford AC, Talley NJ, Schoenfeld PS, et al. (2009) Efficacy of antidepressants and psychological therapies in irritable bowel syndrome: systematic review and meta-analysis. Gut 58, 367-378.

16. Bohn L, Storsrud S, Tornblom H, et al. (2013) Self-reported food-related gastrointestinal symptoms in IBS are common and associated with more severe symptoms and reduced quality of life. Am J Gastroenterol 108, 634-641.

17. Parker HL, Tucker E, Hoad CL, et al. (2016) Development and validation of a large, modular test meal with liquid and solid components for assessment of gastric motor and sensory function by non-invasive imaging. Neurogastroenterol Motil 28, 554-568.

18. Rao SS, Camilleri M, Hasler WL, et al. (2011) Evaluation of gastrointestinal transit in clinical practice: position paper of the American and European Neurogastroenterology and Motility Societies. Neurogastroenterol Motil 23, 8-23.

19. Lewis SJ \& Heaton KW (1997) Stool form scale as a useful guide to intestinal transit time. Scand J Gastroenterol 32, 920-924.

20. Aziz Q, Andersson JL, Valind S, et al. (1997) Identification of human brain loci processing esophageal sensation using positron emission tomography. Gastroenterology 113, 50-59.

21. Parker HL, Tucker E, Blackshaw E, et al. (2017) Clinical assessment of gastric emptying and sensory function utilizing gamma scintigraphy: establishment of reference intervals for the liquid and solid components of the Nottingham test meal in healthy subjects. Neurogastroenterol Motil 29, e13122.

22. Kwiatek MA, Menne D, Steingoetter A, et al. (2009) Effect of meal volume and calorie load on postprandial gastric function and emptying: studies under physiological conditions by combined fiber-optic pressure measurement and MRI. Am J Physiol Gastrointest Liver Physiol 297, G894-G901.

23. Heinrich H, Goetze O, Menne D, et al. (2010) Effect on gastric function and symptoms of drinking wine, black tea, or schnapps with a Swiss cheese fondue: randomised controlled crossover trial. BMJ 341, c6731.

24. Goetze O, Steingoetter A, Menne D, et al. (2007) The effect of macronutrients on gastric volume responses and gastric emptying in humans: a magnetic resonance imaging study. Am J Physiol Gastrointest Liver Physiol 292, G11-G17.

25. Feinle C, Meier O, Otto B, et al. (2001) Role of duodenal lipid and cholecystokinin A receptors in the pathophysiology of functional dyspepsia. Gut 48, 347-355.

26. Simren M, Abrahamsson H \& Bjornsson ES (2007) Lipidinduced colonic hypersensitivity in the irritable bowel syndrome: the role of bowel habit, sex, and psychologic factors. Clin Gastroenterol Hepatol 5, 201-208.

27. Marciani L, Cox EF, Pritchard SE, et al. (2015) Additive effects of gastric volumes and macronutrient composition on the sensation of postprandial fullness in humans. Eur J Clin Nutr 69, 380-384.

28. Parker HL, Curcic J, Heinrich H, et al. (2017) What to eat and drink in the festive season: a pan-European, observational, cross-sectional study. Eur J Gastroenterol Hepatol 29, 608-614.

29. Fox M, Barr C, Nolan S, et al. (2007) The effects of dietary fat and calorie density on esophageal acid exposure and reflux symptoms. Clin Gastroenterol Hepatol 5, 439-444.

30. Rao SS, Welcher K, Zimmerman B, et al. (1998) Is coffee a colonic stimulant? Eur J Gastroenterol Hepatol 10, 113-118.

31. Boekema PJ, Samsom M, van Berge Henegouwen GP, et al. (1999) Coffee and gastrointestinal function: facts and fiction. A review. Scand J Gastroenterol Suppl 230, 35-39.

32. Schuier M, Sies H, Illek B, et al. (2005) Cocoa-related flavonoids inhibit CFTR-mediated chloride transport across T84 human colon epithelia. J Nutr 135, 2320-2325. 
33. Strat KM, Rowley TJ, Smithson AT, et al. (2016) Mechanisms by which cocoa flavanols improve metabolic syndrome and related disorders. J Nutr Biochem 35, 1-21.

34. Sarkar A, Harty S, Lehto SM, et al. (2018) The microbiome in psychology and cognitive neuroscience. Trends Cogn Sci 22, 611-636.

35. Schroeter H, Heiss C, Balzer J, et al. (2006) (-)-Epicatechin mediates beneficial effects of flavanol-rich cocoa on vascular function in humans. Proc Natl Acad Sci US A $\mathbf{1 0 3}$, 1024-1029.

36. Sorond FA, Lipsitz LA, Hollenberg NK, et al. (2008) Cerebral blood flow response to flavanol-rich cocoa in healthy elderly humans. Neuropsychiatr Dis Treat 4, 433-440.

37. Scholey AB, French SJ, Morris PJ, et al. (2010) Consumption of cocoa flavanols results in acute improvements in mood and cognitive performance during sustained mental effort. $J$ Psychopharmacol 24, 1505-1514.
38. Field DT, Williams CM \& Butler LT (2011) Consumption of cocoa flavanols results in an acute improvement in visual and cognitive functions. Physiol Behav 103, 255-260.

39. Karabay A, Saija JD, Field DT, et al. (2018) The acute effects of cocoa flavanols on temporal and spatial attention. Psychopharmacology (Berl) 235, 1497-1511.

40. Francis ST, Head K, Morris PG, et al. (2006) The effect of flavanol-rich cocoa on the fMRI response to a cognitive task in healthy young people. J Cardiovasc Pharmacol 47, Suppl. 2, S215-S220.

41. Canavan C, West J \& Card T (2014) The epidemiology of irritable bowel syndrome. Clin Epidemiol 6, 71-80.

42. Kaplan MA, Prior MJ, Ash RR, et al. (1999) Loperamidesimethicone vs loperamide alone, simethicone alone, and placebo in the treatment of acute diarrhea with gas-related abdominal discomfort. A randomized controlled trial. Arch Fam Med 8, 243-248. 